\title{
Band electron spectrum and thermodynamic properties of the pseudospin-electron model with tunneling splitting of levels
}

\author{
O.Ya.Farenyuk, A.M.Shvaika \\ Institute for Condensed Matter Physics, \\ 1 Svientsitskii Str., 79011 Lviv, Ukraine
}

Received September 27, 2005, in final form February 27, 2006

\begin{abstract}
The pseudospin-electron model with tunneling splitting of levels is considered. Generalization of dynamic mean-field method for systems with correlated hopping was applied to the investigation of the model. Electron spectra, electron concentrations, average values of pseudospins and grand canonical potential were calculated within the alloy-analogy approximation. Electron spectrum and dependencies of the electron concentrations on chemical potential were obtained. It was shown that in the alloy-analogy approximation, the model possesses the first order phase transition to ferromagnetic state with the change of chemical potential and the second order phase transition with the change of temperature.
\end{abstract}

Key words: pseudospin-electron model, correlated hopping, DMFT, ferromagnetism

PACS: $71.10 . \mathrm{Fd}$

\section{Introduction}

One of the main models for describing strongly correlated electron systems is the Hubbard model and its extensions and modifications. It was first proposed as the model describing ferromagnetism in metals. The model is often generalized by introducing additional degrees of freedom. In particular, it is supposed that interaction of electrons with lattice vibrations is important in describing high-temperature superconductors [1] and proton-electron interaction in molecular and crystalline systems with hydrogen bonds [2]. Since anharmonicity in such systems is principally local, it can be considered by using pseudospin formalism. Local potential with two minima, which corresponds to absolute pseudospin value $1 / 2$, appears to be an important particular case of anharmonic vibrations.

The pseudospin-electron model (PEM) [1] turns out to be generalization of the Hubbard model which includes pseudospins with absolute value $1 / 2$. The Hamiltonian of the model has the following form:

$$
\begin{aligned}
& H=\sum_{i} H_{i}+H_{\mathrm{t}}+H_{\mathrm{ss}}, \\
& H_{\mathrm{t}}=\sum_{i, j, \sigma} t_{i j} a_{i \sigma}^{+} a_{j \sigma}, \\
& H_{\mathrm{ss}}=-\frac{1}{2} \sum_{i, j} J_{i j} S_{i}^{z} S_{j}^{z}, \\
& H_{i}=U n_{i \uparrow} n_{i \downarrow}+g n_{i} S_{i}^{z}-h S_{i}^{z}-\Omega S_{i}^{x}-\mu n_{i},
\end{aligned}
$$

where $H_{i}$ is the single-site Hamiltonian, $H_{t}$ is electron transfer and $H_{s s}$ is direct pseudospinpseudospin interaction. $n_{i \sigma}=a_{i \sigma}^{+} a_{j \sigma}$ is the $\sigma$-spin electron number operator, $g n_{i} S_{i}^{z}$ represents interaction with the anharmonic mode (pseudospin). $U$ is Coulomb repulsion of the electrons at one site. $\Omega S_{i}^{x}$ describes the tunneling splitting of vibration mode and $h S_{i}^{z}$ is asymmetry of the anharmonic potential. 
PEM was investigated in many special cases. Among them are: a model with the inclusion of the direct pseudospin-pseudospin interaction (but without electron transfer $\left(t_{i j}=0\right)$ ) $[3,4]$; PEM without the direct pseudospin-pseudospin interaction $\left(J_{i j}=0\right)$, when pseudospins interact through the electron subsystem [5].

Special attention was paid to electron spectra [6], the pseudospin and collective dynamics [7], effective electron-electron interaction [8], correlation functions $\left(\left\langle S^{z} S^{z}\right\rangle,\left\langle S^{z} n\right\rangle,\langle n n\rangle\right)$, and possibility of phase separation and charge-ordered phases [5]. Phase transitions between states with different electron concentrations and with different pseudospin orientations were also studied.

In the case of direct pseudospin-pseudospin interaction, PEM is investigated mainly within the mean field approximation (MFA) [3] and the transfer matrixes formalism [4]. PEM without direct pseudospin-pseudospin is investigated using generalized random phase approximation (GRPA) [9] and dynamic mean-field theory (DMFT) [10]. Many special cases of such modification of PEM were studied: simplified PEM with $U=0$ and $\Omega=0$ or $\Omega \neq 0[7,11]$; a model with the infinitely large Coloumb interaction $U \rightarrow \infty[12,13]$; PEM with $\Omega \neq 0$ but with symmetric anharmonic potential $h=0$ [6]; two-sublattice PEM [14].

The simplified PEM $(U=0$ and $\Omega=0)$ corresponds to the Falicov-Kimball $(\mathrm{FK})$ model but differ in thermodynamic equilibrium conditions $\left(S^{z}=\right.$ const for the FK model and $h=$ const for the PEM) [10].

This paper presents investigation of the PEM with tunneling splitting of the levels without direct pseudospin-pseudospin interaction. Generalization of the dynamic mean-field method for the systems with correlated hopping [15] was applied to the investigation of the model. Within the alloy-analogy approximation, the numerical investigations were conducted. Electron spectrum and dependencies of the electron concentrations on chemical potential were obtained.

Special attention was paid to the phase transition to the ferromagnetic state with the change of chemical potential and temperature. Different aspects of a possible ferromagnetism in PEM model were analyzed.

\section{Analysis of the Hamiltonian}

In the case of narrow bands $(t \ll U)$ and the absence of the direct pseudospin-pseudospin interaction, the single-site Hamiltonian (1.4) plays a role of an initial approximation, so it is useful to introduce the Hubbard operators $X_{i}^{R S} \equiv|i, R\rangle\langle i, S|$ acting in the space spanned by the state vectors, defined as: $|i, p\rangle=\left|n_{i \uparrow}, n_{i \downarrow}, S_{i}^{z}\right\rangle$. Then, single-site Hamiltonian $H_{i}$ can be expressed as [16]:

$$
H_{i}=\sum_{p=1}^{4} \bar{\lambda}_{p} X_{i}^{p p}-\frac{\Omega}{2} \sum_{p=1}^{4}\left(X_{i}^{p \tilde{p}}+X_{i}^{\tilde{p} p}\right)
$$

where $\bar{\lambda}_{1, \tilde{1}}=\mp h / 2, \bar{\lambda}_{2, \tilde{2}}=-2 \mu+U \pm(g-h / 2), \bar{\lambda}_{3, \tilde{3}}=\bar{\lambda}_{4, \tilde{4}}=-\mu \pm(g-h)$.

This Hamiltonian is diagonal for $\Omega=0$ (no tunneling splitting of the vibrational modes). For $\Omega \neq 0$ we can diagonalize it using the following unitary transformation [16]:

$$
\left(\begin{array}{c}
|R\rangle \\
|\tilde{R}\rangle
\end{array}\right)=\left(\begin{array}{cc}
\cos \phi_{r} & \sin \phi_{r} \\
-\sin \phi_{r} & \cos \phi_{r}
\end{array}\right)\left(\begin{array}{c}
|r\rangle \\
|\tilde{r}\rangle
\end{array}\right), \cos \left(2 \phi_{r}\right)=\frac{n_{r} g-h}{\sqrt{\left(n_{r} g-h\right)^{2}+\Omega^{2}}}
$$

Then we have:

$$
H_{i}=\sum_{p} \lambda_{p} X_{i}^{p p}, \quad \lambda_{r, \tilde{r}}=U \delta_{r, 2}-\mu n_{r} \pm \frac{1}{2} \sqrt{\left(n_{r} g-h\right)^{2}+\Omega^{2}} .
$$

Here $n_{r}$ denotes the number of electrons for the state $r: n_{1}=0, n_{2}=2, n_{3}=1, n_{4}=1 ; n_{r}=n_{\tilde{r}}$. In a new basis total Hamiltonian can be written as:

$$
H=\sum_{i} \sum_{r} \lambda_{r} X_{i}^{r r}+\sum_{i j} \sum_{\sigma} t_{i j \sigma} a_{i \sigma}^{+} a_{j \sigma}, \quad a_{i \sigma}=\sum_{m n} A_{m n}^{\sigma} X_{i}^{n m}
$$


where

$$
\begin{aligned}
& A_{41}^{\uparrow}=A_{\tilde{4} \tilde{1}}^{\uparrow}=\cos \phi_{41}, \quad A_{\tilde{4} 1}^{\uparrow}=-A_{4 \tilde{1}}^{\uparrow}=\sin \phi_{41}, \\
& A_{23}^{\uparrow}=A_{\tilde{2} \tilde{3}}^{\uparrow}=\cos \phi_{23}, \quad A_{\tilde{2} 3}^{\uparrow}=-A_{2 \tilde{3}}^{\uparrow}=\sin \phi_{23}, \\
& A_{31}^{\downarrow}=A_{\tilde{3} \tilde{1}}^{\downarrow}=\cos \phi_{31}, \quad A_{\tilde{3} 1}^{\downarrow}=-A_{3 \tilde{1}}^{\downarrow}=\sin \phi_{31}, \\
& A_{24}^{\downarrow}=A_{\tilde{2} \tilde{4}}^{\perp}=-\cos \phi_{24}, \quad A_{\tilde{2} 4}^{\perp}=-A_{2 \tilde{4}}^{\perp}=-\sin \phi_{24},
\end{aligned}
$$

and all the others are equal to zero. Here the designation $\phi_{p q}=\phi_{p}-\phi_{q}$ is used.

One can see that for diagonalized Hamiltonian, the hopping integral value depends on the states of neighboring lattice sites. Hopping of that kind is called correlated hopping and can be dealt with by the use of matrix representations for all quantities [15]. In our case, hopping term can be rewritten in the following way:

$$
t_{i j \sigma} a_{i \sigma}^{+} a_{j \sigma}=\left(\left(Y_{i \tau(\sigma)}^{1}\right)^{+},\left(Y_{i \tau(\sigma)}^{2}\right)^{+},\left(Y_{i \tau(\sigma)}^{3}\right)^{+},\left(Y_{i \tau(\sigma)}^{4}\right)^{+}\right) \hat{t}_{i j \sigma}\left(\begin{array}{c}
Y_{i \tau(\sigma)}^{1} \\
Y_{i \tau(\sigma)}^{2} \\
Y_{i \tau(\sigma)}^{3} \\
Y_{i \tau(\sigma)}^{4}
\end{array}\right),
$$

where:

$$
\begin{array}{llll}
Y_{i \tau(\uparrow)}^{1}=X_{i \tau}^{14}+X_{i \tau}^{\tilde{1} \tilde{4}}, & Y_{i \tau(\uparrow)}^{2}=X_{i \tau}^{1 \tilde{4}}-X_{i \tau}^{\tilde{1} 4}, & Y_{i \tau(\uparrow)}^{3}=X_{i \tau}^{32}+X_{i \tau}^{\tilde{3} \tilde{2}}, & Y_{i \tau(\uparrow)}^{4}=X_{i \tau}^{3 \tilde{2}}-X_{i \tau}^{\tilde{3} 2}, \\
Y_{i \tau(\downarrow)}^{1}=X_{i \tau}^{13}+X_{i \tau}^{\tilde{1} \tilde{3}}, & Y_{i \tau(\downarrow)}^{2}=X_{i \tau}^{1 \tilde{2}}-X_{i \tau}^{\tilde{1} 3}, & Y_{i \tau(\downarrow)}^{3}=X_{i \tau}^{42}+X_{i \tau}^{\tilde{\tilde{\tau}} \tilde{2}}, & Y_{i \tau(\downarrow)}^{4}=X_{i \tau}^{4 \tilde{\tau}}-X_{i \tau}^{\tilde{\tilde{i}} 2}
\end{array}
$$

Here the designation $X_{i \tau}^{p q} \equiv X_{i}^{p q}(\tau)$ was used. Correlated hopping matrix is:

$$
\begin{gathered}
\hat{t}_{i j \sigma}=t_{i j}\left(\gamma_{\sigma}^{T} \otimes \gamma_{\sigma}\right), \\
\gamma_{\uparrow}=\left(\cos \phi_{41}, \sin \phi_{41}, \cos \phi_{23}, \sin \phi_{23}\right), \quad \gamma_{\downarrow}=\left(\cos \phi_{31}, \sin \phi_{31},-\cos \phi_{24},-\sin \phi_{24}\right) .
\end{gathered}
$$

\section{Temperature Green functions. Perturbation theory in terms of electronic hopping}

Investigation of the model is performed using temperature Green functions. Single-site Hamiltonian (1.4) was selected as a zero-order Hamiltonian. Statistical operator can be written as:

$$
\hat{\rho}=\mathrm{e}^{-\beta \hat{H}_{0}} \hat{\sigma}(\beta),
$$

where

$$
\hat{\sigma}(\beta)=T \exp \left\{-\int_{0}^{\beta} \mathrm{d} \tau \int_{0}^{\beta} \mathrm{d} \tau^{\prime} \sum_{i j \sigma} t_{i j \sigma}\left(\tau-\tau^{\prime}\right) a_{i \sigma}^{+}(\tau) a_{j \sigma}\left(\tau^{\prime}\right)\right\}, \quad H_{0}=\sum_{i} H_{i} .
$$

For the grand canonical potential functional we have:

$$
\Omega=-\frac{1}{\beta} \ln \operatorname{Sp} \hat{\rho}=\Omega_{0}-\frac{1}{\beta} \ln \langle\hat{\sigma}(\beta)\rangle_{0}, \quad \Omega_{0}=-\frac{1}{\beta} \ln \operatorname{Sp~}^{-\beta H_{0}} .
$$

So we can define single-electron Green's function as:

$$
G_{i j \sigma}\left(\tau-\tau^{\prime}\right)=-\left\langle T a_{i \sigma}(\tau) a_{j \sigma}^{+}\left(\tau^{\prime}\right)\right\rangle=\frac{\delta \Omega}{\delta t_{j i \sigma}\left(\tau^{\prime}-\tau\right)} .
$$

Let us introduce the corresponding matrix Green functions and other quantities related to the correlated hopping [15]. According to (2.6) and (2.7) matrix form of Green's function $\hat{G}_{i j, \sigma}\left(\tau-\tau^{\prime}\right)$ is defined as:

$$
G_{i j, \sigma}^{\mu \nu}\left(\tau-\tau^{\prime}\right)=\beta \frac{\delta \Omega}{\delta t_{i j, \sigma}^{\nu \mu}\left(\tau-\tau^{\prime}\right)},
$$


where $\mu, \nu$ are matrix indices. Then, the total Green's function (3.4) is equal to:

$$
G_{\sigma}=\operatorname{Sp}\left(\frac{\mathrm{d} \hat{t}_{\sigma}}{\mathrm{d} t_{\sigma}} \hat{G}_{\sigma}\right)=\operatorname{Sp}\left(\left(\gamma_{\sigma}^{T} \otimes \gamma_{\sigma}\right) \hat{G}_{\sigma}\right)=\gamma_{\sigma} \hat{G}_{\sigma} \gamma_{\sigma}^{T}
$$

According to (2.6) and using (3.5) we can write the elements of the $4 \times 4$ matrix Green's function:

$$
G_{i j, \sigma}^{\mu \nu}\left(\tau-\tau^{\prime}\right)=-\left\langle T_{\tau} Y_{i \tau(\sigma)}^{\mu}\left(Y_{j \tau^{\prime}(\sigma)}^{\nu}\right)^{+}\right\rangle
$$

It is obvious that for each matrix element of Green's function we shall have the sum of Tproducts of Hubbard operators like $\left\langle T_{\tau} X_{i}^{p q}(\tau) X_{i^{\prime}}^{p^{\prime} q^{\prime}}\left(\tau^{\prime}\right) X_{i_{1}}^{p_{1} q_{1}}\left(\tau_{1}\right) X_{i_{1}^{\prime}}^{p_{1}^{\prime} q_{1}^{\prime}}\left(\tau_{1}^{\prime}\right) \ldots\right\rangle_{0}$. We can calculate them by consecutive pairing according to the corresponding Wick's theorem [17].

\section{Dynamic mean field theory of correlated hopping}

Since only limited number of lattice models can be solved exactly, one must use some approximations to describe the model behavior. Dynamic Mean-Field Theory (DMFT), which is exact in the limit of infinite spatial dimensions $d \rightarrow \infty$, is one of the most popular approaches [18]. The DMFT is based on the local (single-site) nature of the self-energy in the limit $d \rightarrow \infty$. But, for systems with correlated hopping, self-energy becomes unlocal [19], so it is necessary to modify the standard DMFT approach. Let us recall that self-energy appears in the Dyson equation for the one-electron Green's function, and represents many-electron interactions, which are taken into account as perturbations. There is another natural approach — perturbation theory over electron hopping $t_{i j \sigma}$. In this case, fundamental equation for the one-electron Green's function is the Larkin equation [15]:

$$
\hat{G}_{\mathbf{k} \sigma}(\omega)=\hat{\Xi}_{\mathbf{k} \sigma}(\omega)+\hat{\Xi}_{\mathbf{k} \sigma}(\omega) \hat{t}_{\mathbf{k} \sigma} \hat{G}_{\mathbf{k} \sigma}(\omega),
$$

where $\Xi_{i j}(\omega)$ is an irreducible part of the Green's function that cannot be divided into parts by cutting one hopping line. It was shown in [20] that $\Xi_{i j}(\omega)$ is local in the $d \rightarrow \infty$ when the hopping integral is scaled $t_{i j} \rightarrow \frac{t_{i j}^{*}}{\sqrt{d}}$ in order to obtain finite density of states: $\Xi_{\sigma}\left(\omega_{n}, \mathbf{k}\right)=\Xi_{\sigma}\left(\omega_{n}\right)$, and this statement is more general than the one concerning the local nature of the self-energy [15].

Such a matrix representation makes it possible to reformulate the DMFT for the systems with correlated hopping in terms of local quantities. Equivalence of the irreducible part $\hat{\Xi}_{\sigma}(\omega)$ for the lattice problem and for the single-impurity problem leads to the following equation for coherent potential $J_{\sigma}(\omega)[15]$ :

$$
\frac{1}{N} \sum_{\mathbf{k}}\left[\hat{\Xi}_{\sigma}^{-1}(\omega)-\hat{t}_{\mathbf{k} \sigma}\right]^{-1}=\left[\hat{\Xi}_{\sigma}^{-1}(\omega)-\hat{J}_{\sigma}(\omega)\right]^{-1}=\hat{G}_{\mathrm{imp}, \sigma}(\omega)
$$

which is the matrix generalization of the Brandt-Mielsch equation [21] for the auxiliary KadanoffBaym field. The right-hand side of (4.2) is the Larkin representation of the single-site Green's function $\hat{G}_{\mathrm{imp}, \sigma}(\omega)$ for the single-impurity problem with statistical operator:

$$
\hat{\rho}=\mathrm{e}^{-\beta \hat{H}_{o}} T \exp \left\{-\int_{0}^{\beta} \mathrm{d} \tau \int_{0}^{\beta} \mathrm{d} \tau^{\prime} \sum_{i j \sigma} J_{\sigma}\left(\tau-\tau^{\prime}\right) a_{\sigma}^{+}(\tau) a_{\sigma}\left(\tau^{\prime}\right)\right\} .
$$
$[15]$ :

The grand canonical potential of the lattice in terms of the quantities for the impurity model

$$
\frac{\Omega_{\text {lat }}}{N}=\Omega_{\text {imp }}-\frac{1}{\beta} \sum_{\nu}\left\{\frac{1}{N} \sum_{\mathbf{k}, \sigma} \ln \operatorname{det}\left[1-\hat{\Xi}_{\sigma}\left(\mathrm{i} \omega_{\nu}\right) \hat{t}_{\mathbf{k} \sigma}\right]-\ln \operatorname{det}\left[1-\hat{\Xi}_{\sigma}\left(\mathrm{i} \omega_{\nu}\right) \hat{J}_{\sigma}\left(\mathrm{i} \omega_{\nu}\right)\right]\right\}
$$


where $\Omega_{\text {imp }}$ is grand canonical potential for the impurity model. $\Omega_{\text {imp }}$ can be calculated by applying Wick's theorem, but now we have averages of the products of diagonal Hubbard operators at the same site, so we can reduce their product to a single Hubbard operator. Finally we get [17]:

$$
\Omega_{\mathrm{imp}}=-\frac{1}{\beta} \ln \sum_{p} \mathrm{e}^{-\beta \Omega_{(p)}},
$$

where $\Omega_{(p)}$ is grand canonical potential for subspace $|p\rangle$. Now we can find single-electron Green's function for impurity model by

$$
\hat{G}_{\mathrm{imp}, \sigma}\left(\omega_{n}\right)=\frac{\delta \Omega_{\mathrm{imp}}}{\delta \hat{J}_{\sigma}\left(\omega_{n}\right)}=\sum_{p} w_{p} \hat{G}_{\sigma(p)}\left(\omega_{n}\right), \quad G_{\sigma(p)}^{\mu \nu}\left(\omega_{n}\right)=\frac{\delta \Omega_{(p)}}{\delta J_{\sigma}^{\nu \mu}\left(\omega_{n}\right)},
$$

where $G_{\sigma(p)}^{\mu \nu}\left(\omega_{n}\right)$ are single-electron Green's functions for subspaces characterized by the statistical weights

$$
w_{p}=\frac{\mathrm{e}^{-\beta \Omega_{(p)}}}{\sum_{q} \mathrm{e}^{-\beta \Omega_{(q)}}} .
$$

As a result, single-site (impurity) problem naturally splits into eight subspaces $|p\rangle=|1\rangle, \ldots,|\tilde{4}\rangle$ (see also [17]). We can also introduce irreducible parts of Green's functions in subspaces $\hat{\Xi}_{\sigma(p)}\left(\omega_{n}\right)$ by

$$
\hat{G}_{\sigma(p)}\left(\omega_{n}\right)=\left[\hat{\Xi}_{\sigma(p)}^{-1}\left(\omega_{n}\right)-\hat{J}_{\sigma}\left(\omega_{n}\right)\right]^{-1}
$$

Since the matrix of electron hopping is a direct product (2.7), one can prove that equality (4.2) conserves after transforming it into a scalar form by the relations:

$$
\hat{t}_{\sigma}=\gamma_{\sigma}^{T} t \gamma_{\sigma} \Longrightarrow \hat{J}_{\sigma}=J_{\sigma} \cdot\left(\gamma_{\sigma}^{T} \otimes \gamma_{\sigma}\right) \Leftrightarrow J_{\sigma}=\gamma_{\sigma}^{T} \hat{J}_{\sigma} \gamma_{\sigma}
$$

The same transformations are also applied to all other matrix quantities, such as Green functions and irreducible parts. As a result we can easily switch to a scalar form and back for all equations. For example, equation (4.2) can be transformed in the following way:

$$
\frac{1}{N} \sum_{\mathbf{k}} \gamma\left[\hat{\Xi}_{\sigma}^{-1}(\omega)-\hat{t}_{\mathbf{k} \sigma}\right]^{-1} \gamma^{T}=\sum_{p} w_{p} \gamma\left[\hat{\Xi}_{\sigma(p)}^{-1}(\omega)-\hat{J}_{\sigma}(\omega)\right]^{-1} \gamma^{T}
$$

Then, for left and right part after using the obvious relation:

$$
\hat{A}\left(\gamma^{T} \otimes \gamma\right) \hat{B}=\hat{A} \gamma^{T} \cdot \gamma \hat{B}
$$

we have:

$$
\begin{aligned}
\gamma\left[\hat{\Xi}_{\sigma}^{-1}-\hat{t}_{\mathbf{k} \sigma}\right]^{-1} \gamma^{T} & =\gamma\left(\hat{\Xi}_{\sigma}+\hat{\Xi}_{\sigma}\left[t_{\mathbf{k} \sigma}\left(\gamma^{T} \otimes \gamma\right)\right] \hat{\Xi}_{\sigma}+\cdots\right) \gamma^{T} \\
& =\left(\gamma \Xi_{\sigma} \gamma^{T}\right)+\left(\gamma \Xi_{\sigma} \gamma^{T}\right) t_{\mathbf{k} \sigma}\left(\gamma \Xi_{\sigma} \gamma^{T}\right)+\cdots=\left[\gamma \hat{\Xi}_{\sigma}^{-1} \gamma^{T}-t_{\mathbf{k} \sigma}\right]^{-1}
\end{aligned}
$$

and

$$
\gamma\left[\hat{\Xi}_{\sigma(p)}^{-1}-\hat{J}_{\sigma}\right]^{-1} \gamma^{T}=\left[\gamma \hat{\Xi}_{\sigma(p)}^{-1} \gamma^{T}-J_{\sigma}\right]^{-1}
$$

Thus, the scalar form of equation (4.2) can be written as:

$$
\frac{1}{N} \sum_{\mathbf{k}}\left[\gamma \hat{\Xi}_{\sigma}^{-1} \gamma^{T}-t_{\mathbf{k} \sigma}\right]^{-1}=\left[\gamma \hat{\Xi}_{\sigma}^{-1} \gamma^{T}-J_{\sigma}\right]^{-1}=\sum_{p} w_{p}\left[\gamma \hat{\Xi}_{\sigma(p)}^{-1} \gamma^{T}-J_{\sigma}\right]^{-1}
$$

This equivalence of representations allows us to use whatever form is more convenient in each case. 


\section{Alloy-analogy approximation. Numerical calculations}

For the pseudospin-electron model with tunneling splitting perturbation theory expansion turns out to be too cumbersome and inconvenient - for example, third order contribution has near 30000 terms. In order to proceed, we used simple alloy-analogy approximation:

$$
\hat{\Xi}_{\sigma(p)}\left(\omega_{n}\right)=\hat{g}_{\sigma(p)}\left(\omega_{n}\right)
$$

Here $\hat{g}_{\sigma(p)}\left(\omega_{n}\right)$ are zero-order matrix Green functions for subspaces. For example, $\hat{g}_{\uparrow(1)}\left(\omega_{n}\right)$ is equal to

$$
\hat{g}_{\uparrow(1)}\left(\omega_{n}\right)=\left(\begin{array}{ccc}
\left(\mathrm{i} \omega_{n}-\lambda_{41}\right)^{-1} & 0 & 0 \\
0 & \left(\mathrm{i} \omega_{n}-\lambda_{\tilde{4} 1}\right)^{-1} & 0 \\
0 & 0 & 00 \\
0 & 0 & 0
\end{array}\right) .
$$

Then, for the grand canonical potentials for subspaces one can obtain [17]:

$$
\Omega_{(p)}=\lambda_{p}-\frac{1}{\beta} \sum_{n \sigma} \ln \operatorname{det}\left(1-\hat{\Xi}_{\sigma(p)}\left(\omega_{n}\right) \hat{J}_{\sigma}\left(\omega_{n}\right)\right) .
$$

A scalar form of equations is more convenient for numerical calculations. Starting from (5.3) and using (4.9), one can obtain grand canonical potential for subspaces, which is expressed in scalar quantities:

$$
\Omega_{(p)}=\lambda_{p}-\frac{1}{\beta} \sum_{n \sigma} \ln \left(1-\Xi_{\sigma(p)}\left(\omega_{n}\right) J_{\sigma}\left(\omega_{n}\right)\right), \quad \Xi_{\sigma(p)}=\gamma_{\sigma} \hat{\Xi}_{\sigma(p)} \gamma_{\sigma}^{T} .
$$

The first step of the numerical analysis is to solve the equation (4.2) for coherent potential, when irreducible part is defined by (5.1). This equation may have more than one solution, but it is rather complex and contains integrations. It is more convenient to introduce an equation for statistical weights $w_{p}$, treating them as self-consistency parameters and then use its solutions to calculate Green functions and coherent potential (see also [22]).

Equation for $w_{p}$ has the following form:

$$
w_{p}=\frac{\exp \left(-\beta \Omega_{(p)}\left(\left\{w_{l}\right\}\right)\right)}{\sum_{q} \exp \left(-\beta \Omega_{(q)}\left(\left\{w_{l^{\prime}}\right\}\right)\right)} .
$$

$\Omega_{(p)}\left(\left\{w_{l}\right\}\right)$ is calculated according to $(5.4)$ using coherent potential $J_{\sigma}(\omega)$ which is obtained by a corresponding iterative algorithm. Green functions can be obtained from the same iterative process.
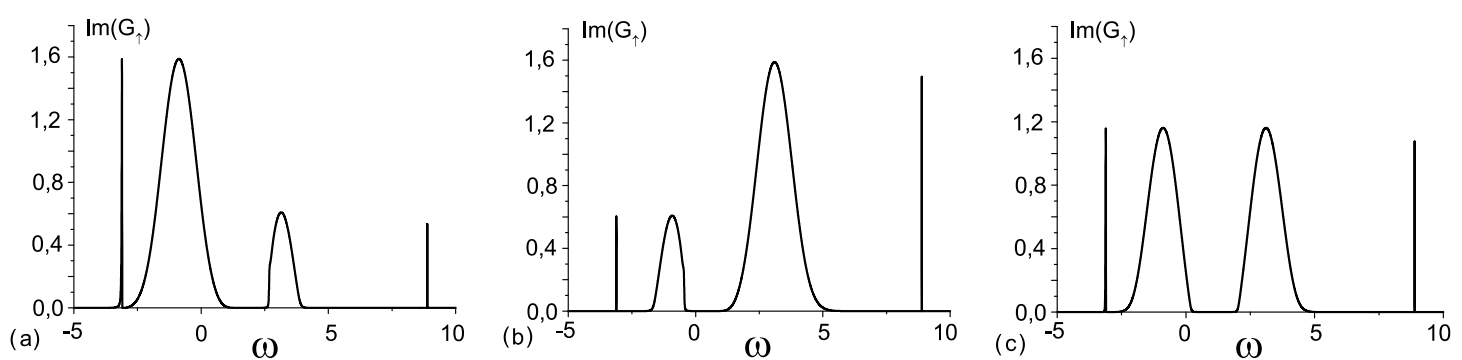

Figure 1. Electron spectrum. Numerical parameters: $h=-2.0, g=1.85, \Omega=1.01, U=4.0$, $\mu=-0.33, T=0.01, t^{*}=1.0,(\mathrm{a}),(\mathrm{b})$ and (c) corresponds to the different solutions, described in table 1.

Initial examination of the solution space of (5.5) was carried out using Multi-Niche Crowding (MNC) variant of the genetic algorithms (GA) [23]. This allowed us to find all possible potential solutions. Then, the candidates were checked and refined using the modified Newton method. 
Table 1. Description of solutions for figure 1. All $w_{p}$, not indicated in the table, are equal to zero.

\begin{tabular}{|c|c|c|c|}
\hline & $\mathrm{a}$ & $\mathrm{b}$ & $\mathrm{c}$ \\
\hline$w_{\tilde{3}}$ & 0.160 & 0.840 & 0.5 \\
$w_{\tilde{4}}$ & 0.840 & 0.160 & 0.5 \\
\hline$n$ & 0.833 & 0.833 & 0.881 \\
$n_{\uparrow}-n_{\downarrow}$ & 0.518 & -0.518 & 0 \\
$S^{z}$ & -0.48 & -0.48 & -0.48 \\
$\Omega_{\text {lat }}$ & -1.95 & -1.95 & -1.93 \\
\hline Ordering & Ferromagnetic & Ferromagnetic & Paramagnetic \\
\hline
\end{tabular}

It has been shown that for rather large $U$, the transition to the ferromagnetic state took place. A typical case is the existence of the three solution sets for self-consistency parameters $w_{p}$ (figure 1). One of them corresponds to the paramagnetic ordering and two others correspond to the ferromagnetic one. Figure 1 shows only the imaginary part of $G_{\uparrow}$. For solution (c), ImG个 and $\operatorname{Im} G_{\downarrow}$ are the same, and $\operatorname{Im} G_{\downarrow}$ for solution (a) is identical to $\operatorname{Im} G_{\uparrow}$ for (b) and vise versa. So, in figure 1 ferromagnetism emerges as a result of redistribution of electron density between bands which corresponds to the $\lambda_{\tilde{4} \tilde{1}}$ and $\lambda_{\tilde{3} \tilde{1}}$ initial single-electron transitions.

The average value of electron concentrations can be obtained in two different ways. One of them is differentiation of the grand canonical potential with respect to the chemical potential $\mu$. The other way is based on using the density of states: $n_{\sigma}=\beta^{-1} \sum_{n} G_{\sigma}\left(\omega_{n}\right)$. For the AA approach, thermodynamically obtained concentrations can get unphysical values for some values of the chemical potential, and thus the second method was used.

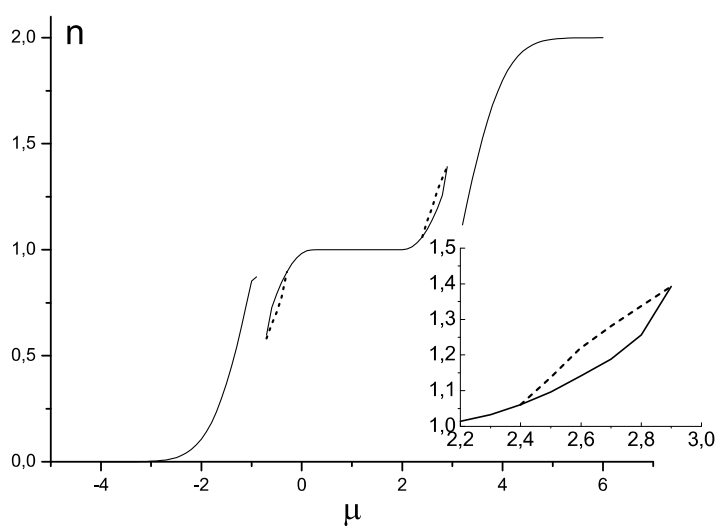

Figure 2. $\mu-n$ diagram. $h=-2.0, g=1.85$, $\Omega=1.01, U=4.0, T=0.01, t^{*}=1.0$. Continuous line corresponds to the paramagnetic ordering and dotted line corresponds to the ferromagnetic ordering.

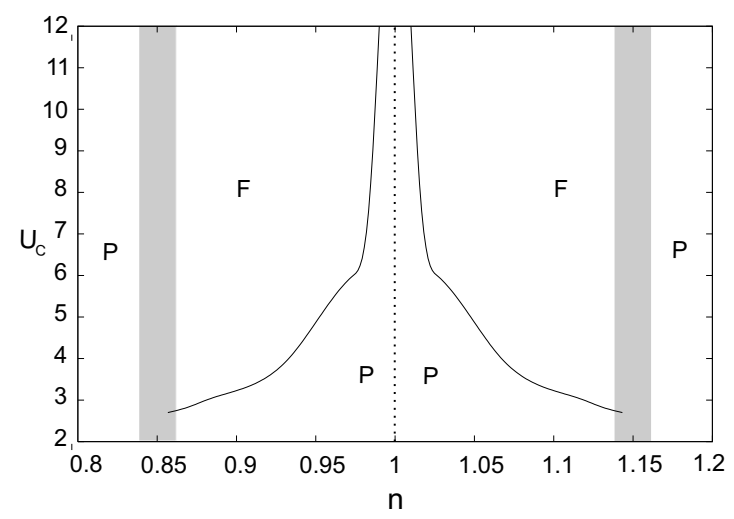

Figure 3. Magnetic phase diagram. F denotes ferromagnetic and $\mathrm{P}$ denotes paramagnetic ordering. Grey color indicates the regions where AA approximation fails. $h=-2.0, g=1.85$, $\Omega=1.01, T=0.01, t^{*}=1.0$.

Figure 2 presents a typical behavior of the electron concentration depending on chemical potential. One can see that ferromagnetism exists for electron concentrations near the half-filling, both for $n<1$ and $n>1$. Gaps on the $\mu-n$ diagram correspond to the unperturbed levels, where the present iterative algorithm of calculations fails for the AA approximation. It is important that ferromagnetism disappears when chemical potential $\mu$ escapes from the bands responsible for it. (They are $\lambda_{\tilde{4} \tilde{1}}$ and $\lambda_{\tilde{3} \tilde{1}}$ in figure 1). Indeed, ferromagnetism is caused by redistribution of electron density between those bands. However, their contribution vanishes rapidly with the change of $\mu$ effectively removing the effect. As a result it is absent for electron concentrations, which substan- 


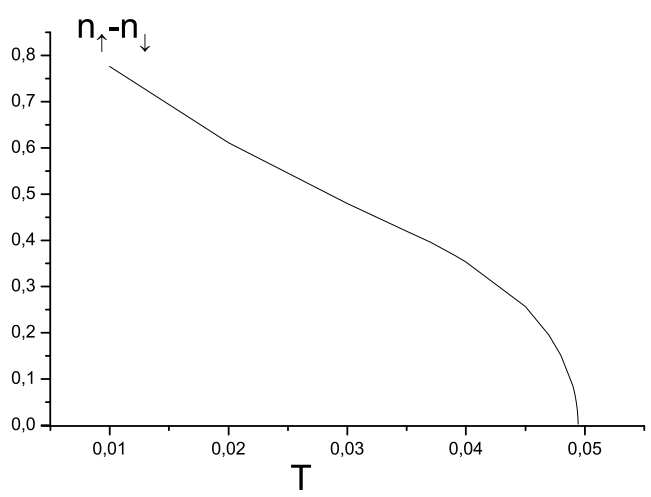

Figure 4. Dependence of magnetization on temperature. $h=-2.0, g=1.85, \Omega=1.01, \mu=1.62$, $U=8.0, t^{*}=1.0$

tially differs from 1 (see also figure 3 ). Thus, critical Coulomb repulsion $U_{c}$ should quickly grow somewhere in the greyed area of the phase diagram presented in figure 3. In our case, the critical Coulomb repulsion $U_{c}$ is much lower than for ordinary Hubbard model [24,25]. Indeed, numerous studies [26-28] revealed that different forms of correlated hopping favour ferromagnetism including the lowering of the critical $U_{c}$ and the stabilization of ferromagnetic ordering. With the change of the temperature, the spectral density is changed, leading to the temperature dependence of magnetization, shown in figure 4.

\section{Conclusions}

The pseudospin-electron model with tunneling splitting of the vibration mode is investigated. It is shown that correlated hopping formalism is useful in investigating the systems with transverse field. Strongly correlated approach for temperature Green functions was applied to the model. Generalization of DMFT for systems with correlated hopping, based on locality of irreducible parts of Green functions in the limit of infinite spatial dimension, was used. It is demonstrated that tunneling-induced correlated hopping can be factorized (2.7), which makes it possible to transform the equations for the single-electron Green functions and grand canonical potential from the matrix to scalar form and vise versa. Alloy-analogy approximation was used in further numerical calculations. Solutions for the symmetry broken phases were distinguished by the weights of subspaces, introduced for the single-side problem, which were considered as a self-consistency parameters. The possibility of ferromagnetism within the alloy-analogy approximation has been shown in contrast to the generally accepted belief that the ferromagnetic phase cannot develop in the AA approximation. The reason for such statements is the fact that AA approximation lacks the mechanism for the spin-dependent shift of the center of gravity of the Hubbard bands [22,29]. However, in recent years other mechanisms of ferromagnetic ordering, where there is no shift in the relative position of the Hubbard bands, were considered [30]. One of them is connected with redistribution of the electron densities between spin-up and spin-down electrons. Our scheme of solving the equations for the coherent potential Green functions leads to the ferromagnetism of this kind. 


\section{References}

1. Müller K.A., Z. Phys. B, 1990, 80, 193.

2. Matsushita E., Phys. Rev. B, 1995, 51, No. 24, 17332.

3. Stasyuk I.V., Havrylyuk Yu., Preprint of the Institute for Condensed Matter Physics, ICMP-98-18E, Lviv, 1998.

4. Farenyuk O.Ya., Shvaika A.M., Preprint of the Institute for Condensed Matter Physics, ICMP-99-21U, Lviv, 1999 (in Ukrainian).

5. Stasyuk I.V., Shvaika A.M., Tabunshchyk K.V., Cond. Matt. Phys., 1999, 2, No. 1(17), 109.

6. Stasyuk I.V., Shvaika A.M., Schachinger, E., Physica C., 1993, 213, 57.

7. Stasyuk I.V., Shvaika A.M., Ferroelectrics, 1997, 192, 1.

8. Stasyuk I.V., Shvaika A.M., Preprint of the Institute for Condensed Matter Physics, ICMP-92-15P, Lviv, 1992.

9. Izyumov, Yu.A., Letfulov, B.M., J. Phys.: Cond. Matter, 1990, 2, 8905.

10. Stasyuk I.V., Shvaika A.M., J. Phys. Studies., 1999, 3, No. 2, 177; Preprint arXiv:cond-mat/9807353, 1998.

11. Stasyuk I.V., Shvaika A.M., Tabunshchyk K.V., Ukrainian Journ. of Phys., 2000, 45, 520; Preprint arXiv:cond-mat/0010334, 2000.

12. Stasyuk I.V., Shvaika A.M., Cond. Matt. Phys., 1994, 3, 134.

13. Stasyuk I.V., Shvaika A.M., Danyliv O.D., Molecular Physics Reports, 1995, 9, 61.

14. O.D.Danyliv, Physica C: Superconductivity 309, 1998, No. 3-4, 303.

15. Shvaika A.M., Phys. Rev. B., 2003, 67, No. 7, 075101; Preprint arXiv:cond-mat/0205322, 2002.

16. Stasyuk I.V., Shvaika A.M., Acta Physica Polonica A, 1993, 84, 293.

17. Shvaika A. M., Phys. Rev. B, 2000, 64, No. 2, 2358.

18. Georges A., Kotliar G., Krauth W., Rozenberg M., Reviews of Modern Physics, 1996, 68, 13.

19. Shiller A., Phys. Rev. B, 1999, 60, 15660.

20. Metzner W., Phys. Rev. B, 1991, 43, 8549.

21. Brandt U., Mielsch C., Z. Phys. B: Condens. Matter, 1989, 75, 365; 1990, 79, 295; 1991, 82, 37.

22. Potthoff M., Herrman T., Wegner T., Nolting W., Phys. Stat. Sol. (b), 1998, 210, 199.

23. Cedeno W., Vemuri V.R., Theoretical Computer Science, 1999, 229, No. 1, 177.

24. Penn D.R., Phys. Rev., 1966, 142, No. 2, 350.

25. Fazekas P., Menge B., Muller-Hartman E., Z. Phys. B, 1990, 78, 69.

26. Vollhardt D., Blümer N., Held K., Kollar M., Schlipf J., Ulmke M., Wahle J., Advances in Solid State Physics, 1999, No. 38, 383; Preprint arXiv:cond-mat/9804112, 1998.

27. Vollhardt D., Blümer N., Held K., Kollar M., Lecture Notes in Physics, Vol. 580, 191, Springer,Heidelberg, 2001; Preprint arXiv:cond-mat/0012203, 2000; Preprint arXiv:cond-mat/0012203, 2000 .

28. Kollar M., Vollhardt D., Phys. Rev. B, 2001, No. 63, 045107; Preprint arXiv:cond-mat/0008015,2000.

29. Schneider J., Drchal V., Phys. Stat. Sol. (b), 1975, 68, 207.

30. Hirsch J.E., Phys. Rev. B, 1999, 59, 6256; Preprint arXiv:cond-mat/9811346, 1998. 


\title{
Електронний спектр та термодинамічні властивості псевдоспін-електронної моделі з тунельним розщепленням рівнів
}

\author{
А.М.Швайка, О.Я.Фаренок \\ Інститут фізики конденсованих систем, \\ вул. Свєнціцького 1, 79011 Львів, Україна \\ Отримано 27 вересня 2005 р., в остаточному вигляді - 27 лютого 2006 р.
}

\begin{abstract}
Розглянуто псевдоспін-електронну модель з тунельним розщепленням рівнів. Для ї̈ дослідження використано узагальнення методу динамічного середнього поля на системи з корельованим переносом. Розрахунок електронної густини станів, концентрації електронів, середнього значення псевдоспінів та великого термодинамічного потенціалу проводився в наближенні типу сплаву. Досліджено залежність електронного спектра та середньої концентрації електронів від хімічного потенціалу. Показано що в наближенні типу сплаву модель демонструє фазовий перехід першого роду у феромагнітний стан при зміні хімічного потенціалу та фазовий перехід другого роду при зміні температури.
\end{abstract}

Ключові слова: псевдоспін-електронна модель, корельований перенос, DMFT, феромагнетизм

PACS: $71.10 . F d$ 